Western University Scholarship@Western

1996

\title{
The Optimal Timing of Procurement Decisions and Patent Allocations
}

Motty Perry

Daniel R. Vincent

Follow this and additional works at: https://ir.lib.uwo.ca/economicsresrpt

Part of the Economics Commons

Citation of this paper:

Perry, Motty, Daniel R. Vincent. "The Optimal Timing of Procurement Decisions and Patent Allocations." Department of Economics Research Reports, 9611. London, ON: Department of Economics, University of Western Ontario (1996). 


\title{
RESEARCH REPORT 9611
}

\section{The Optimal Timing of Procurement Decisions} and Patent Allocations

by

Motty Perry and Daniel R. Vincent

ECONOMICS REFEREASE CFTRE

MAR - 61997

UWIVERSITY of WESTEA UPTARE

October 1996

\author{
Department of Economics \\ Social Science Centre \\ University of Western Ontario \\ London, Ontario, Canada \\ N6A 5C2 \\ econref@sscl.uwo.ca
}


October, 1996

\title{
The Optimal Timing of Procurement Decisions and Patent Allocations
}

by

\author{
Motty Perry ${ }^{*}$
}

and

Daniel R. Vincent ${ }^{* *}$

We illustrate by means of a dynamic research and development race that, while at some points in the race, social incentives and private incentives may coincide, at other points they may diverge -- too many researchers remain in the race. If the social planner cannot determine what stage the researchers have achieved. this informational constraint poses difficulties in ensuring a socially optimal outcome. We show that there is a mechanism which allows the planner to exploit the researchers' private information to determine when and to whom to allocate the exclusive rights to pursue the final prize. This mechanism does not require any transfer of resources and, therefore, will not distort earlier incentives to invest. Furthermore, it is solvable by the iterative elimination of dominated strategies. 


\section{Introduction}

In research and development races, social incentives and private incentives may often diverge -- the private pursuit of profit can induce too many researchers to remain in the race. Of course, this possibility depends on the environment. If the costs of independent research are not too high and the social rewards great, it may be desirable to allow multiple paths of research. We illustrate that both phenomena can occur within the same dynamic research and development race and that, as a result, a non-trivial social planning problem arises. The decision to grant the sole rights to pursue a prize may depend not only on where the leader is but also on his position relative to his rival. The decision is complicated even further if the social planner cannot observe what stage the researchers have achieved. We show that if at least the researchers are aware of their relative positions there is a mechanism which allows the planner to exploit their private information in order to determine when and to whom to allocate the exclusive rights to pursue the final prize. This mechanism does not require any transfer of resources and, therefore, will not distort earlier incentives to invest as would the use of a price mechanism. Furthermore, it is simply implemented by only a few rounds of elimination of dominated strategies.

\section{A Motivating Example}

When the US government decided in the spring of 1942 to commit substantial resources to develop the atomic bomb, it remained unresolved which would be the best source of fissionable material. The expectation was that either separation of the U-235 
uranium isotope or the manufacture of the eiement plutonium would yield fuel for the bomb but there were numerous avenues that could be explored, none of which guaranteed certainty of success. ${ }^{1}$ In the end, the commander of the Manhattan Project, Leslie Groves, authorized four separate pilot projects to be pursued: in Chicago, at Berkeley, Columbia, and the Naval Research Laboratories. ${ }^{2}$ A review committee was created to assess the progress of each venture and decide which would be the most promising method. This decision was evidently not an easy one. Even after Fermi demonstrated the feasibility of using a controlled chain reaction to produce plutonium. the Chicago team was not confident that the decision would go their way. Arthur Compton, the team's leader wrote a letter to Conant arguing that the committee (more specifically, the representatives of Dupont who were to put the process into production) tended to be too pessimistic about the possibilities of their approach and hinted very strongly that perhaps General Electric or Westinghouse should instead be asked to develop plutonium. This implicit threat may or may not have had an effect. In any case, it was decided to implement Fermi's approach to produce fissionable material as well as an additional method. $^{3}$

This episode illustrates the main features of the dynamic incentive problem we analyze. A number of researchers are available to pursue a commonly valued, stochastic prize. Although effective participation requires the investment of some sunk costs, a social surplus-based criterion indicates that, because of the urgency of the task or the great uncertainty of success, it is worthwhile, initially, to invite more than one agent to participate in the race. However, at some later point, that is not observable to a social 
planner, social efficiency may require that only one agent remain to complete the race. The private value to the researchers of winning the prize implies that the principal can not rely on private incentives to induce the optimal set of investigators to drop out of the race even when one of them has made substantially more progress than the rest. In many cases, it will be very costly or even impossible for a social planner to determine when laggard agents should be forced out and even which of them should be the ones to leave the race. Although the review committee did succeed in establishing to its satisfaction that the Fermi approach was superior, Compton's letter suggests that the issue was not an easy one and perhaps even that it was one open to manipulation. Of course, even if the choice of approaches was the correct one, the timing of the decision could easily have been different. Throughout 1942, significant economic and intellectual resources were devoted to a variety of pursuits that might have been more productively focussed on a single path.

The difficulty inherent in making the determination of when and to whom to allocate the exclusive rights to pursue a prize is the focus of this paper. We construct an idealized model of a stochastic race for a prize and show that there exist situations where it is socially efficient to invite many participants at the initial phase but to restrict participation at later phases. The need for a later restriction creates an implementation problem if private incentives alone do not induce the appropriate participants to drop out. In this environment, we show that even if the principal cannot tell when a researcher is far enough ahead to grant him the sole rights to continue the race, as long as the competitors themseives are aware of each other's position, there exists a simple mechanism which can ensure that the optimal decision is made at the desired time without 
distorting the initial incentives to invest.

As suggested by the motivating example, this model can be interpreted as a solution to a private or public procurement problem or a patent race. ${ }^{4}$ For concreteness, we focus on the example of private firms pursuing the development of an innovation in an economy where a benevolent social planner has the authority to limit their participation. Consider an invention which can only be acquired after researchers pass through two or more stages. Firms that reach stages sooner have an advantage over laggard firms. Once a single firm moves sufficiently ahead of the rest, it may be socially desirable to grant that firm the exclusive rights to continue the project. Nevertheless, the attractiveness of the final objective may continue to induce all firms to stay in the race. One goal of an efficient patent system should be to discourage such socially inefficient but privately profitable pursuits. The social planner may then find herself in the position of having to decide whether there is a sufficient asymmetry across researchers to justify the endowment of a patent and to decide to which firm the patent is to be awarded. Very often this decision will have to be made in the absence of any concrete evidence in support of the ruling and or in the absence of an affordable measure for ranking the firms in question. ${ }^{5}$ Even if such investigations are technically feasible, society often will need to rely on an agent such as a patent officer to make the determination for it. The complexity and subjectivity of these decisions make it very difficult for society to monitor its own agent and this creates a situation ripe with the potential for corruption. A system that does not yield such an agent either too much responsibility or too much autonomy to make the decision is needed. 


\section{The Social Problem}

Consider the following dynamic social allocation problem. Two firms seek to develop a product that has a known monetary value $V$. The development of the product requires passage through separate stages. Let $X$ denote the set of such stages. The movement from stage to stage occurs consecutively and stochastically, and is controlled by the investment of research resources period by period. Time has value in that earlier discovery is preferred to later discovery. Players attempt to maximize their discounted expected profits and, in the absence of government intervention, select strategies which are best responses to each other in every period -- that is, they select strategies which are subgame perfect Nash equilibria. The stage that each player has attained is assumed to be common knowledge between the players but not observable by an outside social planner. While the common knowledge assumption is strong, note that it is common to many analyses of R\&D races. ${ }^{6}$ While firms may not enjoy the precise knowledge that their rivals have acquired, they may well be aware of the stage of progress achieved by their rivals. The assumption could also be interpreted as a consequence of the degree of technical sophistication required to assess firms' true positions. Specialists who are well versed in the research area may be able to evaluate quite readily the achievements of both themselves and their rivals. A less well-informed government official may not be able to conduct this evaluation at reasonable cost even though she has access to the same observable data.

In a typical stochastic $R \& D$ race in stages, the closer the researcher is to the final stage, the higher is his expected profits from the race. This monotonicity implies that a 
stage in the R\&D race can be represented by the expected payoff of being at that location in the absence of any competition. For convenience, we choose this representation. The set $X$ is a discrete set of real numbers. Higher values correspond to stages which are closer to the ultimate goal. Let $x_{j}$ denote the stage attained by firm $j$. The social value of restricting the number or firms engaged in research will depend, in general, not only on the absolute position of a firm but also on its position relative to its rival. The "social choice" function, $f: X \rightarrow \mathbb{R}$, is introduced to capture this feature and can be interpreted as follows. Suppose firm $j$ is at stage $x_{j}$. Only if it is at least $f\left(x_{j}\right)$ ahead of firm $i$, is it socially desirable to force $i$ to drop out of the race. The function $f(\bullet)$ allows for the possibility that the distance a firm needs to be leading its rival can vary depending how close it is to the final prize. ${ }^{7}$ We wish to find an allocation mechanism with the following properties:

i) for any firm, $j$, in any period, if $x_{j} \geq x_{i}+f\left(x_{j}\right)$, the mechanism allocates to $j$ the exclusive rights to continue to pursue the prize, otherwise, both firms are allowed to remain;

ii) in equilibrium, no monetary transfers are required from either firm to make the allocation;

iii) the mechanism relies only on the information reported by the two firms in the race;

iv) the mechanism can be implemented by a small number of rounds of iterative elimination of dominated strategies. 
Condition iv) is required to ensure the practicality of the institution. It constrains the designer to select a mechanism that is simple for the firms to understand and play appropriately. The more transparent the mechanism (and the fewer the orders of elimination required), the more plausible it will be that the equilibrium will indeed be played.

Condition iii) is motivated by the recognition that an official who is not directly involved in the race will typically be at a severe informational disadvantage or, alternatively, may not be trusted to make an autonomous decision. There are many situations where the social planner cannot observe the actual positions of the researchers and must rely only on their (self-interested) reports. For example, many of the initial stages of a research program are either unpatentable because of the nature of patent law (US patent law does not allow the granting of patents for the discovery of abstract principles, algorithms or laws of nature) or because the cost of patenting exceeds the potential benefits to the innovator. ${ }^{8}$

The restriction on monetary transfers (condition ii)) stems from the assumption that agents must sink research expenditures initially. If they anticipate having to pay additional costs when their research venture is successful in order to convince an authority to force their slower rival out of the race, the initial incentives to invest may be weakened. ${ }^{9}$ Condition i) stems from the potential inconsistency between social goals and private incentives. Researchers who are maximizing private expected utility may not voluntarily leave the race when the social planner (whose preferences are represented by $f(\bullet)$ ) would like them to. Although it is known that privately induced patent races can 
result in greater than efficient investment (see, for example, Loury, (1979) or Reinganum, (1982)), we are not aware of any models showing that a divergence of social and private incentives can occur at different stages of the race if privately acquired knowledge is not transferable. ${ }^{10}$ In the next section, we construct a simple model of an $R \& D$ race. In this race, whether or not private incentives differ from social incentives depends on which stage the players have attained. This phenomenon implies a simple but non-trivial structure for the social welfare function, $f(\bullet)$, and illustrates the need for an allocation mechanism with these features.

\section{A Research and Development Race}

In this section we use a simple model to show that rational behavior on the part of firms as well as the social planner can create the type of dilemma illustrated in the introduction. Consider the class of environments with the following features: there are two firms, each of which maximizes its expected utility (profits) by entering the race and staying in it until the end even if it falls behind: the social optimum calls for both firms to stay in the race only as long as they are symmetric, and for one firm to quit the race once the other is ahead.

Two firms begin a race in an initial stage 1 . Time is measured in periods. In any period, a firm may move out of one stage to another stochastically as long as it invests in an R\&D cost in that period. ${ }^{11}$ The passage from one stage to another is independent of the actions or position of their rivals. There are three stages. Once either or both firms move out of stage 2 to stage 3 and acquire the prize, $V$, the game ends but the number of periods that the firms may potentially invest in R\&D is infinite. Firms discount the 
future at a common discount factor. $\delta$, per period. For simplicity, we assume that the costs of R\&D, $c$, is the same whether the firm is in stage 1 or 2 . Similarly, the probability of exiting either stage is the same, denoted by $p .^{12}$ If a firm is in stage 2 and successfully develops the innovation alone it earns the full value of the product immediately and the laggard firm earns nothing. If both firms pass out of stage 2 by simultaneously making the discovery, then they split the prize equally. The ex post payoff of a firm is the discounted value of this discovery (if it makes the discovery) minus the discounted R\&D expenditures. Firms maximize expected payoffs given the actions of their rival. The social value of the research and development race is the discounted expected value of the discovery minus the discounted expected costs of both firms.

\section{Private Incentives:}

We begin by calculating backward, from the end of the game, the expected payoffs of the firms in different stages of the race. Denote by $U_{s t}$ the expected payoff for a firm in stage $s$ when its rival is in stage $t$. Thus the continuation value for a firm when both firms in stage 2 and both choose to invest is

$$
U_{22}=p(1-p) V+p^{2}\left(\frac{V}{2}\right)+(1-p)^{2} \delta U_{22}-c=\frac{\frac{V}{2}\left(2 p-p^{2}\right)-c}{1-\delta(1-p)^{2}}
$$

Similarly, the expected payoff for a firm in the second stage when its rival is still in the first stage is 


$$
U_{21}=p V+(1-p) \delta\left[p U_{22}-(1-p) U_{21}\right]-c=\frac{p V+p(1-p) \delta U_{22}-c}{1-\delta(1-p)^{2}} .
$$

$U_{21}>U_{22}--$ it is better to be alone in the final stage than with a rival. Continuing along the same line,

$$
U_{12}=\delta\left[(1-p) p U_{22}+(1-p)^{2} U_{12}\right]-c=\frac{U_{22} \delta(1-p) p-c}{1-\delta(1-p)^{2}} .
$$

Since

$$
\frac{\delta(1-p) p}{1-\delta(1-p)^{2}}<1
$$

it follows that $U_{12}<U_{22}<U_{21}$.

We are interested in races in which a firm which finds itself behind still wishes to remain in the race, that is, where $U_{12}>0$. This implies that the cost of research must be relatively small so that $c \leq \delta p(1-p) U_{22}$ or

$$
c \leq \frac{\delta p^{2}(1-p) V(1-p / 2)}{(1+\delta(1-p))\left(1-\delta(1-p)^{2}\right)}
$$

Inequality (1) (which also implies $U_{22}>0$ ) is the binding constraint in the analysis which follows. Note that for any $\delta>0$, there exist $c, V$ and $p$ all strictly positive for which (1) holds.

We can now evaluate the expected payoff for a firm from entering the race when its rival is in a symmetric position. 


$$
\begin{aligned}
U_{11} & =\delta\left[p(1-p) U_{21}-(1-p) p U_{12}+p^{2} U_{22}+(1-p)^{2} U_{11}\right]-c \\
& =\frac{\delta\left[p(1-p) U_{21}+(1-p) p U_{12}+p^{2} U_{22}\right]-c}{1-\delta(1-p)^{2}}
\end{aligned} .
$$

Since $U_{21}>U_{22}>0$, (2) implies that $U_{11}>U_{12}>0$ if (1) holds. Therefore, as long as inequality (1) holds, it is always a dominant strategy for each firm to remain in the race.

Welfare Analysis:

Let $W_{s t}$ denote the gross welfare generated by having two firms compete, one at stage $s$ and the other at stage $t$. Recall that $W_{s}$ is the welfare generated from having only one firm in the race at stage $s$. Note that our measure of social surplus implies that $W_{s}$ is the same as the private value of being the only firm in the race. Also, since we represent each stage, $s$, by $W_{s}, X$ is $\left\{W_{1}, W_{2}\right\}$. Employing the same approach used to calculate private payoffs,

$$
\begin{aligned}
& W_{22}=\left[p^{2}+2 p(1-p)\right] V \div \delta(1-p)^{2} W_{22}-2 c=\frac{V p(2-p)-2 c}{1-\delta(1-p)^{2}}=2 U_{22}, \\
& W_{2}=p V+\delta(1-p) W_{2}-c=\frac{p V-c}{1-\delta(1-p)}, \\
& W_{12}=p V+\delta(1-p)\left[p W_{22}+(1-p) W_{12}\right]-2 c=\frac{p V-c+2 \delta(1-p) p U_{22}-c}{1-\delta(1-p)^{2}} .
\end{aligned}
$$

and

$$
W_{1}=p \delta W_{2}-(1-p) \delta W_{1}-c=\frac{p \delta W_{2}-c}{1-\delta(1-p)} .
$$

Finally we derive $W_{l l}$. For future use, the calculation is performed under the assumption that if only one firm advances to the second stage then the other firm is 
forced to quit. This yields

$$
W_{11}=\delta\left[p^{2} W_{22}+2 p(1-p) W_{2} \div(1-p)^{2} W_{11}\right]-2 c=\frac{\delta\left[p^{2} W_{2=} \cdot 2 p(1-p) W_{2}\right]-2 c}{-\delta(1-p)^{2}}
$$

The following lemma illustrates the potential conflict between the private motives of the firms and the objective of maximizing the joint welfare of the firms ex ante. It establishes that it may be optimal from a social standpoint to have both firms start the race, and once one is ahead, to force the one that is behind to quit.

Lemma 1: If $\delta$ is small enough, then there exist values $c, p$, and $V$ for which $0<U_{12}$ (inequality (I) is satisfied), yet $W_{22}>W_{2}>W_{12}$, and $W_{11}>W_{1}$.

Proof: See appendix.

Thus, at least for small discount factors, there are values of $c, p$, and $V$ for which a conflict is generated between private and social incentives in the race for the prize. In this example, social preferences can be represented by the function $f(\bullet)$ such that $f\left(W_{l}\right)$ $=\infty$ and $f\left(W_{2}\right)=\Delta$. where $\Delta=W_{2}-W_{1}$. No firm in stage 1 should ever be awarded the patent right and a firm in stage 2 should only be awarded the right if its rival is in stage 1. The proof of Lemma 1 suggests either that the costs of $R \& D$ be small or the urgency of achieving the prize be great (or equivalently, the length of time between periods be large). ${ }^{13}$ The intuition for this is that the social benefit from having more than one firm stems from the effect that more R\&D speeds the expected discovery. The cost arises from the possibility that (ex post) duplicative $R \& D$ occurs. As a result, for values of $\delta$ close to one, there is generally never any social value to having more than one firm in the race. 
Notice that this example is easily modified to illustrate a more complex implementation problem. With more stages, a social planner must be concerned not only with the question of to whom to allocate the sole right to remain in the race but must also determine the correct time, if ever, to make the allocation. For example, suppose that stage 1 was preceded by an earlier stage, call it stage 0 . Let the probability of moving from stage 0 to stage 1 also be $p$ but suppose the investment cost for this period are very low or zero. Lemma 1 can be extended to show that if $U_{12}>0, W_{22}>W_{2}>W_{12}$, and $W_{11}>W_{1}$ then we also have $W_{2}>W_{02}, W_{01}>W_{1}$ and $W_{00}>W_{0}$. Because early research costs are low, it is best to allow both firms to remain in the race even if one firm has moved ahead to stage 1 . However, it still may be desirable to restrict the race once a firm is ahead and in stage 2. From the perspective of the social planner, it must be determined not only if one firm is ahead but also whether the leading firm has achieved stage 2 before forcing the other firm out of the race.

This additional timing problem illustrates why it is not optimal for a social planner to conduct an auction in order to allocate the rights to be the sole firm remaining in the race. The policymaker cannot observe whether a firm has move ahead to stage 2 and, since a leading firm will always wish to win the auction, cannot rely on the participants to initiate an auction-like mechanism at the right time. A further problem with the use of a price mechanism is that in forcing a transfer of wealth at some later stage, it reduces the initial value of the enterprise and therefore may discourage entry at the outset. A mechanism such as cut and choose, since it does not extract resources from the two agents together, might relax this distortion. However, in order not to weaken incentives 
to pursue the prize in the first stage, the mechanism must yield all the surplus to the firm which is in the lead. There is no obvious way to ensure this. In the next section we present an institution that implements the socially efficient outcome and is free from these weaknesses.

\section{An Optimal Mechanism}

The environment described in the previous section indicates the need for a mechanism which can extract enough information from participants in a patent race so as to enable an ill-informed social planner to allocate the patent to a firm if and only if it moves sufficiently ahead of its rival. The mechanism should allow for a relatively flexible definition of what "sufficiently ahead" means, and should not distort the firms' incentives to invest -- the mechanism, in equilibrium, should impose no cost to any participant. It is also important that the optimal strategies in the mechanism will not be too difficult for participants to compute.

In the proposition in this section, we show that a mechanism exists that satisfies all of these criteria. The implementation of the mechanism we present requires that $f(\bullet)$ be nonincreasing as was the case for the example. This appears to be a natural restriction -- the closer to the final prize a given firm finds itself, the smaller the advantage demanded in order to award it the patent. Recall that $X$ is the set of possible locations in the R\&D race. The proposition also requires that $X$ be discrete. If $X$ has the cardinality of the continuum, a similar proof can be employed, however, a tie-breaking assumption concerning the action of agents when they are just indifferent is required. The function, $f(\bullet)$ would also have to be restricted to be continuous in that case. We focus here on the 
discrete case.

To define the mechanism, we will need to extend $f(\bullet)$ so that it is defined over the real interval $\left[W_{1}, W_{n}\right]$. Since the proposition restricts attention to $f(\bullet)$ 's which are nonincreasing, this extension also applies only to nonincreasing $f(\bullet)^{\prime}$ s. Let the extended function take the same values as the original function at all $W_{s}$, and for every interval, $\left[W_{s}, W_{s+1}\right]$, let the extended function be real-valued, continuous and nonincreasing. We use the same notation for this extended function. The mechanism below implements $f(\bullet)$ in three rounds of elimination of weakly dominated strategies:

(i) In each time period, first firm 1 and then firm 2, chooses whether to announce "I am ahead" or not. If neither of them announces, then nothing happens in that period. If only one announces, then the mechanism proceeds to (ii). If both firm announce, then only 1's announcement counts, and the mechanism proceeds to (ii).

(ii) Let firm $j$ be the announcer. Firm $i$ then either "agrees" or "challenges" by calling a value $\tilde{r} \in P$, where $\mathcal{P}=\left\{r_{n}\right\}_{n=1}^{N}, r_{n} \in \mathbb{R}, r_{n}<$ $r_{n+l}$. If firm $i$ chooses to "agree", then firm $j$ is awarded the patent, and the mechanism ends. If firm $i$ challenges, then $j$ pays a fine $P>0$, and the mechanism proceeds to (iii).

(iii) Firm $j$ chooses between buying the patent or not at price $\tilde{r}$. If $j$ chooses to buy, then he gets the patent for a price of $\tilde{r}$ (to be paid to the planner). Firm $i$ then pays a fine $Q>0$. If $j$ chooses not to buy, then firm $i$ buys at $\tilde{r}-f(\tilde{r})$.

End. 
Notice that the mechanism is determined partly by the set of allowable challenges, $P$, a discrete, ordered collection of real numbers. Define $\varepsilon(P)$ to be the greatest distance separating any two adjacent elements of $P$.

Proposition: Suppose $f(\bullet)$ is nonincreasing. For any $X$ which is finite, there is a mechanism with allowable offers, $\mathcal{P}$, which implements $f(x)$ in three rounds of elimination of weakly dominated strategies.

Proof of Proposition: Without loss of generality, we assume that firm $j$ is the proclaimed leader, while firm $i$ is the challenger.

Round 1: For firm j, eliminate every strategy such that given the firm's (true) expected value $v_{j}$, and the price $\tilde{r}$, announced by its rival, firm $j$ chooses to buy when $\tilde{r}>v_{j}$ or chooses not to buy when $\tilde{r}<v_{j}$.

That these strategies are dominated is obvious.

Round 2: a) Eliminate all strategies for firm $i$ in which he challenges when $v_{j} f\left(v_{j}\right) \geq v_{i}$.

This strategy is dominated by not challenging. If $i$ challenges with $\tilde{r}>v_{j} \geq f\left(v_{j}\right)$ $+v_{i}$ then, given the elimination from Round 1 , firm $j$ will never accept and firm $i$ will be forced to buy at price $\tilde{r}-f(\tilde{x})$. This yields utility $v_{i}-\tilde{r}+f(\tilde{r})<-f\left(v_{j}\right)+f(\tilde{r})$ (by definition of $\tilde{\mathrm{r}}$ ) which is less than or equal to zero since $f(\bullet)$ is nonincreasing. If $i$ challenges with $\tilde{r}<v_{j}, j$ will accept and $i$ will have to pay the fine and gain negative utility. If $v_{j}$ is an element of the grid then $i$ challenging with $\tilde{r}=v_{j}$ is weakly dominated by not challenging since if $j$ rejects, $i$ receives the good for zero surplus or negative 
surplus, $v_{i}-f\left(v_{j}\right)$ but if $j$ accepts $i$ pays the fine and receives a strictly negative payoff, $-Q$.

b) Also eliminate all strategies in which he does not challenge, when $v_{j}-f\left(v_{j}\right)<v_{i}$.

Define $r_{m}\left(v_{j}\right)$ to be the minimum number on the grid that is strictly greater that $v_{j}$. There are two cases to consider.

Case 1: There exists $\tilde{r}>v_{j}$ such that $\tilde{r}-f(\tilde{r})<v_{i}$ and $v_{j}$ is not an element of the grid. Clearly, for $\varepsilon(\odot)$ small enough, challenging with $r_{m}\left(v_{j}\right)$ dominates all other strategies.

Case 2: As in case 1 but $v_{j}$ is an element in the grid. Then every point in the grid above $r_{m}\left(v_{j}\right)$ is dominated by $r_{m}\left(v_{j}\right)$ as is any point below $v_{j}$. Finally not challenging is dominated by challenging with $r_{m}\left(v_{j}\right)$. It follows that if $\varepsilon(P)$ is small enough, player $i$ has 2 strategies left: $(\alpha)$ challenge with $r_{m}\left(v_{j}\right) ;(\beta)$ challenge with $v_{j}$.

Round 3: Eliminate all remaining strategies for $j$ except those in which he claims to be ahead when his expected value is $v_{j}$, his opponent's value is $v_{i}$ and $v_{j}-f\left(v_{j}\right) \geq v_{i}$.

The elimination in this round is obvious except possibly for the situation described in Round 2(b), Case 2. In that case, Round 2 removes all strategies except two for firm $i$. However, either of those strategies involve a challenge from $i$ which yields $j$ a strictly negative payoff.

In equilibrium, then, a leading firm will only claim to be ahead when $v_{j} \geq v_{i}+$ $f\left(v_{j}\right)$, as desired. The follower will not challenge and therefore the leader will be awarded the right to remain with no payment. 


\section{Conclusions}

An $R \& D$ race is often inodeled as one with a well defined finishing line where the first to reach that line gets the exclusive rights to pursue the final prize. Where exactly to put the finishing line, can also be an important policy decision. In discussing this policy variable, it is usually implicitly assumed in the literature, that references can be made only to the leader's position in the R\&D race. (Such is a policy stating that exclusive rights should be awarded to the first to reach a certain stage.) The optimal rule, however, depends not only on the leader's stage of research, but also on the location of its rivals. If the follower is very close to the leader then his investment might not be socially wasteful even in a relatively late stage of the $R \& D$. At the same time, a big jump ahead by one of the firms in an early stage of the race, could make further investments of lagging firms inefficient. One of the reasons for ignoring the location of the slower firm (by the literature as well as policy-makers) is of course an observability problem. One can force the leader to file for a patent (say), but it is much more difficult to get the follower to reveal his position. Our mechanism provides incentives for the follower to report exactly when the leading firm is not far enough ahead.

Our approach may be thought of as an extension of the research on the optimal design of patents to the issue of the timing of patent allocations. Recently, a U.S. research company, Human Genome Sciences, generated controversy by attempting to acquire patents for partial gene sequences. the functions of which were not well known at the time. (The Washington Post, April 17, 1996). The R\&D model we described illustrates a situation where such an award would be socially desirable. In addition, we present a 
very simple mechanism which would enable a policymaker to implement the optimal allocation rule if and only if the leading firm has made enough progress relative to the location of its rivals. An attractive feature of the mechanism is that while the leading firm reveals its location truthfully, it does so without the need to reveal details of the innovation. Furthermore, because the mechanism does not require the decisionmaker to have full information, it eliminates the need for this agent to bear the costs of evaluation. As a result, it also reduces the opportunities for corruption by the officers who must implement the social decision. 


\section{Appendix}

Proof of Lemma 1: We start by showing the following claim.

Claim: For every $\delta>0$, there exist values $c$. and $V$ for which $U_{I 2}>0$ (i.e. inequality (1) is satisfied), yet $W_{2}>W_{12}$.

$$
\begin{aligned}
W_{12} & =\frac{p V-c+2 \delta(1-p) p U_{22}-c}{1-\delta(1-p)^{2}} \\
& =\frac{p V-c}{1-\delta(1-p)} \frac{1-\delta(1-p)}{1-\delta(1-p)^{2}}+\frac{\delta(1-p) p U_{22}-c}{1}-\delta(1-p)^{2}+\frac{\delta(1-p) p U_{22}}{1-\delta(1-p)^{2}} \\
& =W_{2}\left[1-\frac{\delta(1-p) p}{1-\delta(1-p)^{2}}\right]+\frac{\delta(1-p) p U_{22}-c}{1-\delta(1-p)^{2}}+\frac{\delta(1-p) p U_{22}}{1-\delta(1-p)^{2}}
\end{aligned}
$$

Recall that we assumed in (1) that $\delta(1-p) p U_{22} \geq c$. So let (1) hold with equality. The third term in the last line above becomes zero and we can write

$$
W_{12}=W_{2}+\frac{\delta(1-p) p}{1-\delta(1-p)^{2}}\left[U_{22}-W_{2}\right]
$$

Note that $W_{2}$ is also the private profit of a firm which finds itself alone in stage 2. Therefore, $U_{22}<W_{2}$ and we have $W_{12}<W_{2}$ for $V, p, c$ such that (1) holds close enough to equality.

We now continue with the proof of Lemma 1.

$$
\begin{aligned}
W_{11} & =\frac{\delta\left[p^{2} W_{22}+2 p(1-p) W_{2}\right]-2 c}{1-\delta(1-p)^{2}} \\
& =\frac{\delta\left(p^{2} W_{22}+p(1-2 p) W_{2}-c\right.}{1-\delta(1-p)^{2}}-\frac{\delta p W_{2}-c}{(1-\delta(1-p))} \frac{(1-\delta(1-p))}{\left(1-\delta(1-p)^{2}\right)} \\
& =\frac{\delta p\left(p\left(W_{22}-W_{2}\right)+(1-p) W_{2}\right)-c}{1-\delta(1-p)^{2}}+W_{1}\left(1-\frac{\delta(1-p) p}{1-\delta(1-p)^{2}}\right) \\
& =W_{1}+\frac{\delta p\left(p\left(W_{22}-W_{2}\right)+(1-p)\left(W_{2}-W_{1}\right)\right)-c}{1-\delta(1-p)^{2}} .
\end{aligned}
$$


So $W_{11}>W_{1}$ iff

$$
\delta p\left(p\left(W_{22}-W_{2}\right)+(1-p)\left(W_{2}-W_{1}\right)\right) \geq c .
$$

Recall that $W_{22}=2 U_{22}$. Setting (1) again as an equality, we get

$$
c=\frac{W_{22}(1-p) p \delta}{2} .
$$

Can $\left(^{*}\right)$ now hold? Substituting and dividing by $\delta$ and $p$ yields

$$
p\left(W_{22}-W_{2}\right)+(1-p)\left(W_{2}-W_{1}-\frac{W_{22}}{2}\right) \geq 0 .
$$

Now express $W_{22}$ and $W_{1}$ in terms of $W_{2}$ :

$$
\begin{aligned}
W_{22} & =\frac{V p-c}{1-\delta(1-p)} \frac{1-\delta(1-p)}{1-\delta(1-p)^{2}}+\frac{V p(1-p)-c}{1-\delta(1-p)^{2}} \\
& =W_{2}+\frac{p(1-p)\left(V-\delta W_{2}\right)-c}{1-\delta(1-p)^{2}}
\end{aligned}
$$

and

$$
W_{1}=\frac{\delta p W_{2}-c}{1-\delta(1-p)}
$$

Since

$$
V-\delta W_{2}=V-\frac{\delta(p V-c)}{1-\delta(1-p)}=\frac{(1-\delta) V+\delta c}{1-\delta(1-p)}
$$

it follows from the way we rewrote $W_{22}$ that $W_{22}>W_{2}$ iff

$$
\frac{p(1-p)((1-\delta) V+\delta c)}{1-\delta(1-p)}>c
$$

or

$$
(1-\delta) p(1-p) V>c[1-\delta(1-p)-\delta p(1-p)]=c\left[1-\delta\left(1-p^{2}\right)\right]
$$


As $\delta \Rightarrow 1$, the above inequality can not hold. However. as $\delta \Rightarrow 0$, it holds iff

$p(I-p) V>c$. Thus, if $\delta$ is small enough and $p(I-p) V>c$. then $W_{22}>W_{2}$. Inequality $\left.{ }^{* *}\right)$

now becomes

$$
\frac{p^{2}(1-p) V-c}{1-\delta(1-p)^{2}}-\frac{\delta p^{2}(1-p) W_{2}}{1-\delta(1-p)^{2}}+(1-p) \frac{(1-\delta) W_{2}+c}{1-\delta(1-p)}-\frac{1-p}{2}\left(W_{2}+\frac{p(1-p)\left(V-\delta W_{2}\right)-c}{1-\delta(1-p)^{2}}\right) \geq 0 .
$$

As $\delta \Rightarrow 0$, the left hand side approaches

$$
\begin{aligned}
& p^{2}(1-p) V-c+(1-p)\left(W_{2}+c\right)-\frac{1-p}{2}\left(W_{2}+p(1-p) V-c\right) \\
& =V\left[p^{2}(1-p)-p \frac{(1-p)^{2}}{2}\right]+\frac{1-p}{2} W_{2}+c\left[(1-p)+\frac{1-p}{2}-1\right] \\
& =V\left[p(1-p)\left(p-\frac{(1-p)}{2}\right)\right]+\frac{1-p}{2} W_{2}+c\left[\frac{1-p}{2}-p\right] . \\
& =\frac{1-p}{2} W_{2}+\frac{1}{2}(3 p-1)[p(1-p) V-c] .
\end{aligned}
$$

Therefore, a sufficient condition for the lemma to hold is for (1) to hold with (almost) equality, for $\delta$ small. for $p \geq 1 / 3$ and $p(1-p) V \geq c$. Note that $p(I-p) V>c$ is exactly the condition we needed for $W_{22}>W_{2}$. 


\section{ENDNOTES}

*Departments of Economics, Hebrew University, Jerusalem, Israel and University of Western Ontario, Social Sciences Centre, London, ON, Canada, N6A 5C2.

${ }^{* *}$ Department of Economics, University of Western Ontario, Social Sciences Centre, London, ON, Canada, N6A 5C2.

1"In a May 14, 1942 memo to [Vannevar] Bush, James Conant stated that there were five separation or production methods which were about equally likely to succeed." Smyth (1945). Bush and Conant were members of the Military Policy Committee which oversaw the Manhattan Project.

${ }^{2}$ The costs of the individual laboratories were large. For example, the Berkeley facility alone required $\$ 30$ million in set-up costs (Groves (1962)) while Conant estimated that the total cost to the pilot plant stage would be more than $\$ 500$ million (Smyth (1945)).

${ }^{3}$ See Groves (1962) and Jones (1985).

${ }^{4}$ Private firms may also wish to encourage multiple paths of research. In its race with BASF to develop a synthetic indigo dye in Germany at the end of the nineteenth century, Hoechst sponsored four separate pilot projects to test differing approaches. (Freeman, 1982).

${ }^{5}$ For example, the costs of assessing the relative merits of designs for US military aircraft are often enormous. Peck and Scherer (1963) estimated that in the early 1960's, the fulltime services of more than 200 engineers over several months were required by the US Airforce to assess proposed aircraft designs.

${ }^{6}$ See, for example, Fudenberg, et al. (1983) or Reinganum (1982). 
${ }^{7}$ If the planner simply wishes to award the prize whenever one firm moves ahead, the King Solomon mechanism suggested by Glazer and Ma (1989) would achieve many of the desired goals. However, typically the optimal time to award an exclusive prize may be a much more complicated function of the firms' positions. The function $f(\bullet)$ here is intended to capture this complexity.

${ }^{8}$ Scotchmer (1991) argues "Patent law requires disclosure for the same reason that innovators dislike it: it is the vehicle by which technical knowledge is passed from the patenting firm to it competitors.[...] As a consequence the innovator could hold the product off the market until it develops the second more valuable generation product."

${ }^{9} \mathrm{~A}$ distortion similar in spirit arises in Green and Scotchmer (1995). They point out that the inability to capture all of the social value of a first stage discovery reduces the initial incentive to invest. On the other hand, if another researcher who makes an incremental improvement on this discovery is forced to pay all of the incremental social surplus to the first stage inventor, the incentives of the later researcher to incur further sunk costs in research are diminished and the second stage may not be developed.

${ }^{10}$ Reinganum (1982) shows that the levels of socially efficient and privately induced $R \& D$ may cross over time if jointly conducted research enables the transmission of knowledge.

${ }^{11}$ The notion that innovation requires the progression through various discrete stages is common in R and D races. Fudenberg, et al. (1983), characterize the distinction as that between the conceptual definition of research program and the actual implementation of it. Riordan and Sappington point out that the procurement of military equipment in the US very often occurs in stages. In the early, design, stages, a number 
of firms compete for the (often) sole rights to provide the products to the government. At some point in this process, government procurement officials must decide which firm wins this valuable right.

${ }^{12}$ It is more reasonable to allow probabilities and costs to vary with the stage achieved. We allow for this in a later discussion of the implications of extending the number of stages but for the purposes of the formal analysis, this is not required and the assumption of similar costs and probabilities eases the notation significantly.

${ }^{13}$ In the memo referred to above, Conant writes "All five methods will be entering very expensive pilot-plant development within the next six months; furthermore, if time is to be saved, the production plants should be under design and construction before the pilot plant is finished. To embark in the Napoleonic approach to the problem would require the commitment of perhaps $\$ 500$ million and quite a mass of machinery. Anything less than this will mean either the abandonment or the slowing down of one of the methods. While all five methods now appear to be about equally promising, clearly the time to production by the five routes will certainly not be the same but might vary by six months or a year. Therefore, if one discards one or two or three of the methods now, one may be betting on the slower horse unconsciously." Smyth (1977). 


\section{REFERENCES}

FREEMAN, Christopher. The Economics of Industrial Innovation. Second Edition, Cambridge: MIT Press, 1982.

FUDENBERG, D., GILBERT, R., STIGLITZ, J. and TIROLE, J. "Preemption, Leapfrogging, and Competition in Patent Races." European Economic Review, 22 (1983), pp. 3-31.

GLAZER, J. and MA, C.-T. "Efficient Allocation of a "Prize" -- King Solomon's Dilemma," Games and Economic Behavior, 1 (1989), pp. 222-233.

GREEN, J. and SCOTCHMER, S. "On the Division of Profit in Sequential Innovation." RAND Journal of Economics, 26(1) (Spring, 1995), pp. 2033.

GROVES, Leslie. Now it can be told: The Story of the Manhattan Project. New York: Harper Brothers, 1962.

JONES, Vincent. Manhattan: The Army and the Atomic Bomb. Washington, DC: Center of Military History, United States Army, 1985.

LOURY G. "Market Structure and Innovation." Quarterly Journal of Economics, 93 (1979), pp. 395-410.

PECK, M. and SCHERER, F. The Weapons Acquisition Process: An Economic Analysis. Cambridge: Harvard University Press, 1962.

REINGANUM, J. "Dynamic Games of Innovation." Journal of Economic Theorv, 25 (1982), pp. 21-41.

RIORDAN, M. and SAPPINGTON, D.E.M. "Second Sourcing." RAND Journal of Economics 20(1) (Spring, 1989), Pp. 41-58.

SCOTCHMER, Suzanne. "Standing on the Shoulders of Giants." Journal of Economic Perspectives, 5 (Winter, 1991), pp. 29-42.

SMYTH, H.D. "A General Account of the Development of Methods of Using Atomic Energy for Military Purposes Under the Auspices of the United States Government. (The Smyth Report, 1945). Reprinted in The secret History of the Atomic Bomb. Anthony Caves Brown and Charles MacDonald (eds.), Vol. 20, No. 1. New York: Delta Books, 1977.

The Washington Post, April 17, 1996. 